\title{
MORTALIDAD PERINATAL EN EL HOSPITAL SAN JUAN DE DIOS, CUCUTA
}

\section{Doctores Alberto Duarte Contreras *, Ramiro Zúñiga Gamboa **,

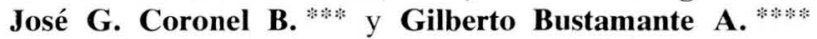

\section{Introducción}

Hemos recopilado en este correlato 4.324 historias clínicas del Servicio de Maternidad del Hospital San Juan de Dios, de Cúcuta.

Representan ellas 4.379 partos, que son la totalidad de los atendidos entre el $1^{\text {o }}$ de octubre de 1960 y el 31 de julio del presente año (1963).

Es nuestro objetivo estudiar la relación que existe en nuestro medio entre Anoxia y Mortalidad perinatal.

\section{Material y métodos}

Las condiciones de trabajo y las contingencias de nuestro servicio de maternidad y del servicio de prematuros y recién nacidos corren parejas con las de todas las instituciones nacionales de beneficencia.

Cuenta nuestro servicio con 25 camas; está atendido por un equipo de cinco médicos, un residente y un interno. Adjunto funciona el servicio de prematuros y recién nacidos, con 10 cunas y 5 incubadoras; en él trabajaban dos pediatras especializados en este ajetreo. En este servicio se examinan todos los nacidos vivos y se retienen los prematuros, más todos aque-llos que aun cuando tengan un peso normal hayan nacido en parto distécico o intervenido, o que presenten problemas de isoinmunización, o los que

* Médico Jefe del Departamento de Obstetricia y Ginecología y Jefe del Servicio de Maternidad del Hospital San Juan de Dios.

** Médico Adjunto del Servicio de Ginecología.

*** Médico Jefe del Servicio de Prematuros y Recién Nacidos y Jefe del Servicio de Medicina Interna de la Clínica Infantil del Hospital San Juan de Dios.

**** Médico Adjunto del Servicio de Prematuros y Recién Nacidos y del Servicio de Medicina Interna de la Clínica Infantil del Hospital San Juan de Dios de Cúcuta.

Trabajo leído ante el V Congreso Colombiano de Obstetricia y Ginecología. Medellín - 1963. 
hayan tenido cualquier dificultad respiratoria al nacer, o un Apgar inferior a 7/10, o los hijos de las toxémicas, o cuantos, a juicio de los pediatras, requieran observación especial. Los demás pasan al lado de sus madres, pues seguimos el método del "rooming in".

Estudiaremos todos los productos obtenidos con un peso superior a los 400 gramos, incluyendo las molas, y todos quedarán bajo la denominación general de parto; este es el motivo para que los porcentajes de mortalidad nos queden visiblemente elevados, pues, repetimos, nuestra intención es analizar el componente anóxico, sacrificando cifras estadísticas favorables.

Emplearemos la clasificación del comité del cuidado materno infantil, del Consejo de Servicios Médicos de la Asociación Médica Americana de 1959 (1).

\section{Resultados}

CUADRO 1

Mortalidad perinatal

Total partos

Nacidos vivos

Prematuros

$\%$ de prematurez $\quad 9.26$

Mortalidad fetal

$\%$ mortalidad fetal

5.54

Antes de 28 semanas

28 semanas o más

De tiempo desconocido

Muerte temprana

$\%$ mortalidad temprana 1.64

Muerte perinatal

$\%$ mortalidad perinatal 7.18
Ai examinar este cuadro nos salta a la vista el alto porcentaje de mortalidad fetal o mortinatalidad, de $5.54 \%$, cuando el porcentaje (2), (3), (4) de la Clínica Primero de Mayo es de

Fundación Virgilio Barco, de Cúcuta

Hospital San José, Bogotá

Instituto Protección Materno Infantil

4.48

Pero no debemos olvidar que hemos estudiado todos los productos obtenidos con un peso de 400 gramos o más, y también hemos incluído las molas (13 en total- $0.29 \%$ ); ello hace que nuestro porcentaje de mortaiidad fetal sea elevado.

En cambio, nuestro porcentaje de mortalidad neonatal temprana es muy satisfactorio: $1.64 \%$, si le comparamos (5) con el de la Clínica

David Restrepo

Fundación Virgilio Barco

Clínica Primero de Mayo

Instituto Protección Materno Infantil

4.3

4.136

383

243

96

135

12

63

311

No obstante tener el hospital una consulta prenatal organizada y existir en Cúcuta 7 Centros de Salud, donde se presta una atención más o menos eficiente, el $87.03 \%$ de las parturientas llegan a nuestro servicio sin haber tenido control prenatal alguno; sólo el 12.97 han tenido control prenatal, y el $5 \%$ acuden después de haber recibido atención de comadronas; muy frecuentemente llegan en período de expulsión. 
CUADRO 2

En relación con control prenatal

\begin{tabular}{lrrrr}
\hline $\begin{array}{l}\text { Control } \\
\text { prenatal }\end{array}$ & \multicolumn{2}{c}{ Partos } & Muerte & $\begin{array}{r}\text { peri- } \\
\text { natal }\end{array}$ \\
& & & & Total \\
\hline & $\%$ & Total & $\%$ \\
\hline Con control & 568 & 12.97 & 17 & 2.9 \\
Sin control & 3.811 & 87.03 & 293 & 7.6 \\
\hline Total & 4.379 & 100.00 & 310 & \\
\hline
\end{tabular}

Vemos también cómo aquellas gestantes que no tuvieron control prenatal rindieron un mayor tributo a la mortalidad perinatal: $7.6 \%$, en tanto que quienes tuvieron asistencia médica prenatal presentaron una mortalidad de $2.9 \%$ (6).

Podemos apreciar que no hubo mortalidad perinatal en gestantes con edad inferior a los 15 años; de aquí en adelante fue aumentando la mortalidad perinatal a medida que las gestantes
CUADRO 3

Mortalidad perinatal en relación con edad de las gestantes.

\begin{tabular}{lrrrr}
\hline Edad & & $\begin{array}{c}\text { Total } \\
\text { partos }\end{array}$ & \multicolumn{2}{c}{$\begin{array}{c}\text { Muertes perinatales } \\
\text { Total }\end{array}$} \\
\hline & & \multicolumn{1}{c}{$\%$} \\
\hline Menos de 15 & 5 & 0 & 0 \\
De 15 a 19 & 797 & 37 & 4.64 \\
De 20 a 29 & 2.236 & 134 & 5.99 \\
De 30 a 34 & 728 & 69 & 9.74 \\
De 35 a 45 & 599 & 60 & 10.01 \\
Más de 45 & 4 & 3 & 75.00 \\
Desconocida & 10 & 8 & 80.00 \\
\hline Total & 4.379 & 311 & 7.18 \\
\hline
\end{tabular}

avanzaban en edad, pero el porcentaje se elevó de manera por demás brusca en las gestaciones mayores de los 45 años.

\section{CUADRO 4}

Mortalidad perinatal en relación con la gravidez.

\begin{tabular}{|c|c|c|c|c|c|c|c|}
\hline \multirow{4}{*}{ Gravidez } & \multicolumn{3}{|l|}{ Total } & \multicolumn{4}{|c|}{ Muertes perinatales } \\
\hline & \multirow{3}{*}{ partos } & \multirow{2}{*}{\multicolumn{2}{|c|}{ Fetal }} & \multirow{2}{*}{\multicolumn{2}{|c|}{ Temprana }} & \multirow{2}{*}{\multicolumn{2}{|c|}{ Perinatal }} \\
\hline & & & & & & & \\
\hline & & No & $\%$ & No & $\%$ & No & $\%$ \\
\hline I & 1.045 & 22 & 2.1 & 23 & 2.2 & 45 & 4.30 \\
\hline II a V & 2.016 & 75 & 3.72 & 30 & 1.48 & 105 & 5.20 \\
\hline VI a X & 914 & 81 & 8.86 & 12 & 1.30 & 93 & 10.16 \\
\hline Más de X & 353 & 60 & 16.99 & 2 & 0.51 & 62 & 17.56 \\
\hline Desconocida & 51 & 5 & & 1 & & 6 & 11.76 \\
\hline Total & 4.379 & 243 & & 68 & & 311 & \\
\hline
\end{tabular}

Vemos cómo la mortalidad perinatal es mayor a medida que aumenta la gravidez, y se acentúa aún más des- pués de la gravidez VI, pero este aumento se hace a expensas de la mortalidad fetal, que va aumentando en 
progresión aritmética, en tanto que la mortalidad temprana disminuye vertiginosamente y de manera sorprendente al aumentar la gravidez (7).

\section{CUADRO 5}

Muerte fetal según su peso en gramos

\begin{tabular}{|c|c|c|}
\hline Peso en gramos & Total & $\%$ \\
\hline 400 a 1.000 & 88 & 36.21 \\
\hline 1.001 a 1.500 & 13 & 5.35 \\
\hline 1.501 a 2.000 & 9 & 3.71 \\
\hline 2.001 a 2.500 & 23 & 9.47 \\
\hline 2.501 a 4.000 & 88 & 36.21 \\
\hline 4.001 a 6.200 & 5 & 2.06 \\
\hline Desconocido & 17 & 6.99 \\
\hline Total & 243 & 100.00 \\
\hline
\end{tabular}

Vemos cómo en nuestra estadística de mortalidad fetal, corren parejas los productos obtenidos por debajo de los 1.000 gramos con los mortinatos nacidos a término y con peso comprendido entre los 2.501 y los 4.000 gramos.

En los de peso inferior a 1.000 gramos, tenemos los hijos de las ges- tantes con enfermedades crónicas, con graves alteraciones nutricionales, con alteraciones funcionales u orgánicas del cuello uterino, con serios disturbios hormonales, etc.

En los de peso comprendido entre 2.500 y 4.000 gramos, encontramos los nacidos de gestantes toxémicas, o con problemas de implantación o de funcionamiento placentario, o con enfermedades agudas evolutivas, y los fetos nacidos con malformaciones o con problemas de isoinmunización, o de partos abandonados, o de uso inadecuado de oxitócicos, o de conductas erróneas.

Las 13 molas obtenidas están incluídas en los de peso desconocido; ellas representan el $0.29 \%$ de la mortalidad perinatal.

Cabe destacar que la mortalidad fetal corresponde, casi en su totalidad, a productos de madres que no tuvieron ningún control prenatal, y que la mayoría de las causas que motivaron esta mortalidad, pudieron ser más o menos prevenibles.

CUADRO 6

Mortalidad perinatal en toxémicas

\begin{tabular}{lrrrrr}
\hline & Total & \multicolumn{3}{c}{ Mortalidad } \\
Madres & partos & Fetal & Neonatal & \multicolumn{2}{c}{ Perinatal } \\
\hline Toxémicas & 97 & 21 & 00 & 21 & 21.64 \\
No toxémicas & 4.282 & 222 & 68 & 290 & 6.77 \\
\hline \multicolumn{1}{c}{ Total } & 4.379 & 243 & 68 & 311 & 7.18 \\
\hline
\end{tabular}

Este cuadro es muy diciente: entre 97 toxémicas (pre eclampticas y eclámticas) tuvimos 21 muertes perinatales, y todas ellas a expensas del factor fetal, con un porcentaje de mortalidad del $21.64 \%$, en tanto que en madres 
no toxémicas el porcentaje fue de $6.77 \%$.

Queremos hacer hincapié en que de las 97 madres toxémicas, únicamente cuatro $(4.12 \%)$ habían tenido control prenatal.
Quiere ello decir que esta mortalidad se podría disminuír en mucho si las gestantes buscasen oportunamente servicios médicos prenatales (8), (9), (10).

\section{CUADRO 7}

Mortalidad neonatal en partos intervenidos

\begin{tabular}{|c|c|c|c|c|c|}
\hline \multirow[b]{2}{*}{ En parto } & \multirow{2}{*}{$\begin{array}{l}\text { nacidos } \\
\text { Total }\end{array}$} & \multirow[b]{2}{*}{$\%$} & \multicolumn{2}{|c|}{$\begin{array}{l}\text { Mortalidad } \\
\text { neonatal }\end{array}$} & $\begin{array}{c}\text { Componente } \\
\text { mortalidad } \\
\text { neonatal }\end{array}$ \\
\hline & & & N? & $\%$ & $\%$ \\
\hline Espontáneo & 3.696 & 89.34 & 60 & 1.61 & 1.45 \\
\hline Intervenido & 440 & 10.66 & 8 & 1.81 & 0.19 \\
\hline Cesárea & 246 & 5.95 & 3 & 1.21 & 0.07 \\
\hline Fórceps & 68 & 1.65 & 0 & & \\
\hline Vacuum & 101 & 2.45 & 2 & 1.98 & 0.05 \\
\hline Extracción podálica & 18 & 0.44 & 3 & 16.66 & 0.07 \\
\hline $\begin{array}{l}\text { Versión interna y } \\
\text { extracción pod. }\end{array}$ & 7 & 0.17 & 0 & & \\
\hline
\end{tabular}

El $89.34 \%$ de nuestras pacientes estudiadas tuvieron parto espontáneo, y en ellas la mortalidad neonatal fue de $1.61 \%$, y su aporte al componente de mortalidad neonatal fue de $1.45 \%$. El $10.66 \%$ tuvo parto intervenido, con un tributo de $1.81 \%$ a la mortalidad neonatal y de $0.19 \%$ al componente de mortalidad neonatal.

Discriminando los partos intervenidos, y en lo que atañe a cesáreas, nuestra mortalidad neonatal temprana está baja: $1.21 \%$ (11), (12). Ello obedece a que nuestro criterio es bien definido. Tratamos de hacer lo más pronto posible una valoración real del trabajo de parto, y si tenemos la seguridad o el presentimiento bien cimentado de que al efectuarse éste por vías naturales va a ser a costa de un trabajo prolongado, o terminado por un forceps, o un vacuum de difícil aplicación, con posible detrimento de la integridad materno-fetal, o estamos ante la inminencia de un sufrimiento fetal, no dudamos en intervenir con cesárea. (Es para nosotros más placentero se nos trate de intervencionistas, que llevar sobre nuestra conciencia muertes neonatales, o, peor aún, secuelas irreversibles de anoxia, que son un baldón para el obstetra y una carga para la familia y para la sociedad).

El alto porcentaje de anomalías neurológicas, psíquicas, y las casi constantes alteraciones electroencefalográficas halladas en los niños por cesáreas tardías debe ser un toque de alarma pa- 
ra el obstetra que en la época actual aún orienta su conducta hacia la práctica sistemática de la operación cesárea tardía (13).

En lo que respecta al forceps, debemos anotar que usamos únicamente los de Simpson y Piper para rotaciones o para extracción de cabeza última retenida. Con él no tuvimos mortalidad perinatal, pero sí muchos destrozos vaginales, vesicales rectales y de cuello uterino.

Desde el mes de agosto de 1961 estamos empleando el vacuum extractor o ventosa de Malström en la totalidad de los partos, con demora en el descenso o en la expulsión. Tuvimos al principio dos muertes neonatales tempranas por gran traumatismo obstétrico atribuíbles exclusivamente al mal empleo de la ventosa; más ahora que hemos adquirido experiencia y confianza en su manejo, hemos tenido completo éxito en los 99 partos en que la hemos utilizado.

La extracción podálica nos da una mortalidad elevada, tal vez porque la hemos practicado tardíamente.

La versión interna la empleamos única y exclusivamente para extracción del segundo feto en partos gemelares, cuando su situación impide el parto espontáneo; en estas condiciones y con una buena técnica no puede darnos mortalidad perinatal.

\section{CUADRO 8}

Muerte neonatal, según edad

\begin{tabular}{lcc}
\hline Edad & Murieron & $\%$ \\
\hline Primer día & 33 & 48.55 \\
Segundo día & 100 & 14.70 \\
Tercer día & 7 & 10.29 \\
Cuarto día & 6 & 8.82 \\
Quinto día & 4 & 5.88 \\
Sexto día & 3 & 4.41 \\
7 a 10 días & 5 & 7.35 \\
\hline Total & 5 & 7.35 \\
\hline
\end{tabular}

Podemos observar que la mortalidad neonatal en las primeras 24 horas es del 48.55\%: durante esto día ocurrieron casi todas las muertes consecutivas a asfixia postnatal, a atelectasias, a inmadurez respiratoria, o sea, son muertes que llevan consigo el componente anóxico.

Las muertes consecutivas a traumatismo obstétrico ocurrieron durante los tres primeros días.

Del tercer día en adelante se presentaron las muertes debidas a malformaciones congénitas, discracias sanguíneas y prematuridad; y entre el sexto y el décimo días las debidas a infecciones. 


\section{CUADRO 9}

Causas de mortalidad perinatal

\begin{tabular}{|c|c|c|c|c|c|}
\hline & Muerte fetal & & & Muerte temprana & \\
\hline & Causa & global & parcial & Causa & $\%$ \\
\hline I. & $\begin{array}{l}\text { Causas y condiciones } \\
\text { maternas }\end{array}$ & 15.64 & & Prematuridad & 49.25 \\
\hline & Enfermedad crónica & 3.29 & 21.05 & Traumatismo & \\
\hline & Toxemia & 8.65 & 55.26 & obstétrico & 11.94 \\
\hline & Otras & 3.70 & 23.69 & $\begin{array}{l}\text { Asfixia postnatal o } \\
\text { atelactasias }\end{array}$ & 14.93 \\
\hline II . & $\begin{array}{l}\text { Causas de feto, } \\
\text { placenta, cordón }\end{array}$ & 47.74 & & Respiratorias & 10.45 \\
\hline & $\begin{array}{l}\text { Desprendimiento pre- } \\
\text { maturo placenta }\end{array}$ & 17.69 & 37.07 & Otras & 13.43 \\
\hline & $\begin{array}{l}\text { Otras alteraciones } \\
\text { cordón, placenta }\end{array}$ & 16.47 & 34.48 & & \\
\hline & $\begin{array}{l}\text { Deformaciones congé- } \\
\text { nitas del feto }\end{array}$ & 1.23 & 2.59 & & \\
\hline & $\begin{array}{l}\text { Eritroblastosis e incom- } \\
\text { patibilidades sanguíneas }\end{array}$ & 0.41 & 0.86 & & \\
\hline & Otras & 11.94 & 25.00 & & \\
\hline III. & Desconocidas & 36.62 & & & \\
\hline
\end{tabular}

Para analizar las causas de mortalidad perinatal, las dividimos en dos grandes capítulos: Causas de muerte fetal y causas de muerte temprana.

\section{A) Causas de muerte fetal.}

Se deben estudiar en tres grupos bien definidos: causas maternas, causas y condiciones de feto, placenta, cordón, y causas desconocidas.

Las causas maternas (38 muertes fetales) nos dan el $15.64 \%$ global. En ellas, las enfermedades crónicas como diabetes mellitus, sífilis, enfermedades renales y cardiovasculares, desnutrición, anemias, parasitosis, etc., alcanzan el $21.05 \%$, en tanto que las toxe- mias ocurren en el $55.26 \%$, o sea: las enfermedades crónicas maternas y las toxemias son las causantes del $76.31 \%$ de las muertes fetales atribuíbles a causa materna.

Estas dos causas influyen como responsables directas de la patología placentaria (desprendimientos, fibrosis, degeneraciones), lo que trae consigo anoxia y muerte fetal.

Otras, como la falta de control de las alteraciones de la contractilidad uterina, el uso inadecuado y el abuso de oxitócicos y de maniobras, etc., nos dan el $23.69 \%$ de las muertes fetales de este grupo. 
Son muertes prevenibles en gran escala si se lleva control prenatal adecuado y oportuno.

Las causas de feto, placenta, cordón (116 muertes fetales) producen el $47.74 \%$ global de mortalidad fetal, y de este grupo los desprendimientos prematuros de placenta dan el $30.07 \%$, y otras alteraciones de cordón y placenta dan el $34.48 \%$, o sea, estas dos dan el $71.55 \%$ de las muertes fetales por causas de feto, placenta, cordón. Las deformaciones congénitas nos dan el 2.59\%; las incompatibilidades $\mathrm{Rh}$, hr y $\mathrm{ABO}$ dan el $0.86 \%$, y otras como infecciones del amnios, molas, etc., nos dan el $25 \%$ de las muertes fetales de este grupo: son muertes prácticamente inevitables.

El porcentaje tan elevado de causa desconocida ( 89 casos $36.62 \%$ ) tiene, sin duda alguna, un fondo morboso agudo o crónico, causante o asociadc a un estado anóxico intrauterino. Estamos convencidos de que en un futuro cercano podremos disminuír este porcentaje ubicando estos casos en grupos etiológicos definidos, ayudados por el examen antomopatológico efectuado como rutina. Ello es tan cierto que en un trabajo preliminar que presentáramos al IV Congreso de Ginecología y Obstetricia de México, en el mes de junio de este año, sobre mortalidad pe- rinatal, dábamos una cifra de $42.4 \%$ para este grupo (14).

B) Causas de muerte temprana.

En el capítulo de muerte temprana encontramos primero la prematurez con 33 casos $(49.25 \%)$. El diagnóstico de prematurez lo hemos hecho a todo producto obtenido con un peso de 2.500 gramos o menos (15).

El traumatismo obstétrico, 8 casos, nos da un porcentaje muy elevado $(11.94 \%)$, y nos obliga a tener un mayor cuidado en la oportuna indicación y correcta técnica en el manejo de las maniobras obstétricas.

La asfixia postnatal y la atelectasia (10 casos) nos dan también un alto índice de mortalidad neonatal temprana, lo mismo que las causas respiratorias ( 7 casos, $10.45 \%$ ); ello nos obliga a revisar, perfeccionar y actualizar nuestros métodos y prácticas de reanimación, a usar de manera más frecuente y oportuna la intubación endotraqueal, seguida de aspiración, el lavado gástrico, la oxígeno y la antibioterapia.

En otras ( 9 casos- $13.43 \%$ ) incluímos las deformaciones congénitas, atresias, y una serie de alteraciones diagnosticadas o no, y que no hemos podido catalogar en ninguno de los grupos estudiados anteriormente. 


\section{CUADRO 10}

Muerte de nacidos vivos, según haya presencia o ausencia de anoxia en madre, feto, placenta o cordón.

\begin{tabular}{lll}
\hline Causa de muerte & Presencia & Ausencia \\
de anoxia & de anoxia \\
\hline
\end{tabular}

Vuerte fetal

Causas y condiciones en la madre

Diabetes mellitus $\mathrm{X}$

Toxemia $\mathrm{X}$

Causas determinantes en feto, placenta y cordón

Prolapso, ruptura del cordón

Placenta previa

Desprendimiento prem. placenta

Anencefalia

Hidrocefalia

Eritroblastosis

Maceración de causa no especificada

$\begin{array}{ll}\mathrm{X} & \\ \mathrm{X} & \\ \mathrm{X} & \mathrm{X} \\ & \mathrm{X}\end{array}$

$\mathrm{X}$

X

\section{Muerte temprana del recién nacido}

Monstruosidades

\begin{tabular}{ll} 
& $\mathrm{X}$ \\
& $\mathrm{X}$ \\
$\mathrm{X}$ & \\
$\mathrm{X}$ & $\mathrm{X}$ \\
\hline
\end{tabular}

Este cuadro es el que nos sirve sencia o ausencia del componente de patrón para agrupar las causas anóxico.

de mortalidad, de acuerdo con la pre- 


\section{CUADRO 11}

Nacidos vivos y muertes fetales entre 4136 partos, de acuerdo con peso y con presencia o ausencia de componente de anoxia.

\begin{tabular}{|c|c|c|c|c|c|c|c|c|c|}
\hline \multirow{3}{*}{$\begin{array}{l}\text { Peso } \\
\text { en } \\
\text { gramos }\end{array}$} & \multicolumn{4}{|c|}{ Muerte fetal } & \multicolumn{5}{|c|}{ Nacidos vivos } \\
\hline & \multirow{2}{*}{ Total } & \multirow{2}{*}{$\begin{array}{c}\text { Con } \\
\text { anoxia }\end{array}$} & \multirow{2}{*}{$\underset{\text { anoxia }}{\text { Sin }}$} & \multirow{2}{*}{ Total } & \multicolumn{4}{|c|}{ Muertes tempranas } & \multirow[b]{2}{*}{$\begin{array}{l}\text { Sobrevi- } \\
\text { a vientes }\end{array}$} \\
\hline & & & & & Total & $\%$ & $\begin{array}{c}\text { Cons } \\
\text { anoxia }\end{array}$ & $\begin{array}{l}\text { Sins } \\
\text { anoxia }\end{array}$ & \\
\hline Total & 243 & 116 & 127 & 4.136 & 68 & 1.64 & 28 & 40 & 4.068 \\
\hline Prematuros & 133 & 72 & 61 & 383 & 61 & 15.90 & 22 & 39 & 322 \\
\hline 400 a 1.000 & 88 & 50 & 38 & 12 & 12 & 100.00 & & 12 & 0 \\
\hline 1.001 a 1.500 & 13 & 7 & 6 & 36 & 19 & 52.77 & 7 & 12 & 17 \\
\hline 1.501 a 2.000 & 9 & 5 & 4 & 103 & 22 & 21.35 & 9 & 13 & 81 \\
\hline 2.001 a 2.500 & 23 & 10 & 13 & 232 & 8 & 3.44 & 6 & 2 & 224 \\
\hline 2.501 a 4.000 & 88 & 33 & 55 & 3.634 & 7 & 0.19 & 6 & 1 & 3.627 \\
\hline-.001 a 6.200 & 5 & 5 & & 87 & & & & & 87 \\
\hline Desconocido & 17 & 6 & 11 & 32 & & & & & 32 \\
\hline
\end{tabular}

En este cuadro presentamos la totalidad de los partos estudiados en nuestro trabajo, agrupándolos de acuerdo con el peso de los productos. Y en esta forma damos en cifras totales, primero, la mortalidad fetal y la contribución que en ella tuvo el componente anóxico. Luego presentamos el total de los nacidos vivos, e indicamos el número de muertes tempranas y la discriminación de esta mortalidad, según haya tenido como causa etiológica la presencia o la ausencia del factor anóxico. En la última casilla encontramos el número de sobrevivientes en cada uno de los grupos estudiados.

Vale decir: nuestro porcentaje global de muertes tempranas es de 1.64. Nuestra mortalidad neonatal temprana en prematuros es de $15.90 \%$, mortalidad muy elevada a ojos vistas: pe- ro debemos recordar que estamos incluyendo productos desde los $400 \mathrm{gms}$, y que entendemos por nacido vivo a todo recién nacido que haya presentado al salir del canal del parto cualquiera de estos signos vitales: pulsación del cordón, latidos cardíacos, movimientos de músculos voluntarios $\mathrm{y} / \mathrm{o}$ movimientos de músculos respiratorios; el cordón ha podido ser ligado o no, y la placenta ha podido estar desprendida o no (18).

La mortalidad de nacidos a término es apenas de $0.19 \%$ y no tenemos mortalidad neonatal temprana en nacidos con peso superior a los 4001 gramos.

En los de peso no especificado, que son todos de peso superior a los 2501 gramos, tampoco tuvimos mortalidad neonatal temprana. 
Tuvimos 92 productos con peso superior a los 4.001 gramos: Cinco mortinatos y 87 nacidos vivos. De éstos, el de peso más alto tuvo 6.200 gramos.

Quisimos conocer la relación que pudiera existir entre estos productos de sobrepeso y la glicemia materna. Cinco gestantes tenían control prenatal con glicemia normal. Practicamos la curva de glicemia a 47 parturientas en el transcurso de las primeras 24 horas del puerperio (16), ante la imposibilidad absoluta de haberlas investigado antes del parto, y encontramos una glicemia superior a la normal solamente en cuatro de ellas. Citamos estas pacientes a nuevos controles: sólo dos asistieron, $\mathrm{y}$ sus glicemias fueron entonces normales (17).

En cuanto a los recién nacidos no observamos síntoma alguno que nos llamase la atención.

\section{CUADRO 12}

Muertes perinatales y porcentaje de mortalidad perinatal, en relación con el peso en gramos y con la presencia o ausencia del componente de anoxia.

\begin{tabular}{|c|c|c|c|c|c|c|c|c|}
\hline $\begin{array}{l}\text { Clasificación } \\
\text { por peso } \\
\text { cn gramos }\end{array}$ & $\begin{array}{l}\text { Total } \\
\text { partos }\end{array}$ & $\begin{array}{r}\text { Mue } \\
\text { Total }\end{array}$ & $\begin{array}{l}\text { Con perin } \\
\text { Con } \\
\text { anoxia }\end{array}$ & $\begin{array}{l}\text { atales } \\
\text { Sin } \\
\text { anoxia }\end{array}$ & $\begin{array}{l}\text { Mortalidad } \\
\text { Con } \\
\text { anoxia }\end{array}$ & $\begin{array}{l}\text { perinata } \\
\text { Sin } \\
\text { anoxia }\end{array}$ & $\begin{array}{l}\text { Sobrevi- } \\
\text { vientes \% }\end{array}$ & $\because$ \\
\hline Total & 4.379 & 311 & 144 & 167 & 3.28 & 3.81 & 92.91 & 46.30 \\
\hline Prematuros & 516 & 194 & 94 & 100 & 37.59 & 18.21 & 44.20 & 48.45 \\
\hline 400 a 1.000 & 100 & 100 & 50 & 50 & 50.00 & 50.00 & 00.00 & 50.00 \\
\hline 1.001 a 1.500 & 49 & 32 & 14 & 18 & 28.57 & 36.73 & 34.70 & 43.68 \\
\hline 1.501 a 2.000 & 112 & 31 & 14 & 17 & 12.50 & 15.17 & 72.33 & 45.16 \\
\hline 2.001 a 2.500 & 255 & 31 & 16 & 15 & 7.11 & 5.88 & 87.01 & 51.61 \\
\hline $2.501 \mathrm{a} 4.000$ & 3.732 & 95 & 39 & 56 & 1.04 & 1.44 & 97.52 & 41.05 \\
\hline 4.001 a 6.200 & 92 & 5 & 5 & & 5.43 & & 94.57 & 100.00 \\
\hline Desconocido & 49 & 17 & 6 & 11 & 12.24 & 22.44 & 65.32 & 35.29 \\
\hline
\end{tabular}

* Componente de anoxia de mortalidad en $\%$ mortalidad perinatal total.

En este cuadro analizamos la mortalidad perinatal glogal, agrupada también de acuerdo con el peso de los productos; y en la primera parte damos las cifras totales de los casos estudiados. En la segunda parte analizamos separadamente el porcentaje de mortalidad perinatal, según haya intervenido en él o no el factor anoxia; damos luego el porcentaje de superviven- cia. Por último anotamos el componente de anoxia de mortalidad en relación con el porcentaje de mortalidad perinatal total.

Observamos claramente la relación tan íntima que existe entre la anoxia como causante de muerte y el peso de los productos. $\mathrm{Y}$ así vemos que el componente anóxico estuvo presente en el $48.45 \%$ de la mortalidad de prema- 
turos; en la de los productos a término lo estuvo en el $41.05 \%$, en tanto que en el de los productos de sobrepeso lo estuvo en el $100 \%$.

El alto porcentaje que hemos obtenido del componente de anoxia como factor etiológico en nuestra mortalidad perinatal nos obliga a tener presente la gravedad de la anoxia, pues aun cuando no siempre es ella causante de mortalidad perinatal, sí está comprometida como factor etiológico de graves desórdenes morbosos, algunos de ellos de pronóstico sombrío, tales como la parálisis cerebral, la epilepsia, los trastornos del lenguaje, los retardos mentales, la pérdida total de la visión o la audición, etc.

\section{CONCLUSIONES}

Es indispensable para lograr disminuír la mortalidad perinatal, adelantar una campaña bien dirigida sobre la necesidad del control prenatal.

\section{RESUMEN}

Se revisan 4.324 Historias Clínicas del Servicio de Maternidad del Hospital San Juan de Dios de Cúcuta.

Se estudia la mortalidad perinatal.

Se dan conclusiones.

\section{BIBLIOGRAFIA}

1. A guide for the study of perinatal mortality and morbidity. J. A. M. A. 1959.

2. CAMACHO GAMBA JORGE: Mortalidad perinatal en Colombia. Rev. Col. Ped. 90. Abril 1958.

3. PLATA RUEDA ERNESTO: Morbilidad perinatal. IV jornadas pediátricas colombianas. Memorias. Cali. 1-18. 1958.

4. FEBRES CORDERO LUCIO: Mortalidad perinatal en la Fundación Virgilio Barco, de Cúcuta. Tres años de labores. Memorias de las IV jornadas pediátricas colombianas. Cali. 64-70. 1958.

5. PLATA RUEDA ERNESTO: Mortalidad neonatal hospitalaria en Bogotá. Rev. Col. Ped. 5: 417-493. 1957.

6. Ochoa Mejía Germán: Mortalidad perinatal. Tesis de grado. Fac. de Med. U. de Antioquia. 1957.

7. AMARIZ MORA ALBERTO: Edad de gestación del recién nacido en la Clínica Primero de Mayo, de Bogotá. Rev. Col. Ped. 3: 173-178. 1961.

8. MENDOZA MARIO: Morbimortalidad del recién nacido de madre toxémica en la maternidad, en Barranquilla. Rev. Col. Ped. 1: 39-45. 1960.

9. GUTIERREZ ALFONSO y Col.: Mortalidad fetal en las toxemias. Rev. Col. Obst. y Gin. 5: Mayo, 1954. 
10. PLATA RUEDA ERNESTO: Consideraciones sobre el recién nacido vivo de la toxémica. Rev. Col. Obst. y Gin. 5: 103. 1954.

11. DELGADO CALDERON ERNESTO: Mortalidad perinatal en la operación cesárea. Rev. Col. Obst. y Gin. 5: 173-187. 1961.

12. RITCHIE D. C.: Maternal and perinatal mortality associated with cesarean section in Alberta (1955-1959). The Canadian Med. Ass. J. 13: 649-655. 1963.

13. ROSENVASSER B.: Porvenir inmediato y alejado de los niños extraídos con operación cesárea tardía. (Estudio clínico, psíquico, y electroencefalográfico). Rev. Col. Obst. y Gin. 2: 81-88. 1953.

14. DUARTE CONTRERAS ALBERTO y Col.: Mortalidad perinatal en el Hospital San Juan de Dios, de Cúcuta (Colombia). Rev. Col. Obst. y Gin. 4: 413-423. 1963.

15. ALBORNOZ MEDINA CARLOS y Col:: Nuevos conceptos de prematuridad en nuestro medio. Rev. Col. Ped. 1: 57-62. 1961.

16. MAIllo ADOLFO: La glucosuria del embarazo. Medicamenta. 384: 212-214. 1963.

17 BARnes P. H.: Prediabetes and pregnacy. The Canadian Med. Ass. J. 7: 301-303. Agosto 17 de 1963.

18. ABRAMSON HAROLD and Col. Resuscitation of the newborn infant. 21-36. 1960. 